\title{
Abandoned Object Detection Using Dual Background Model from Surveillance Videos
}

\author{
Purvi Bhandari $^{\# 1}$, Tushar Ratanpara ${ }^{\# 2}$, Dr. K. H. Wandra ${ }^{\# 3}$ \\ ${ }^{\# 1}$ Department of Computer Engineering, Dharmsinh Desai University, Nadiad, India \\ \#2 Assistant Professor, CE Department, Dharmsinh Desai University, Nadiad, India \\ ${ }^{\# 3}$ Dean, Faculty of Technology and Engineering, C U Shah University, Wadhwan City, India \\ ${ }^{1}$ bhandaripurvi@gmail.com \\ 2tushar.ratanpara@gmail.com \\ ${ }^{3}$ khwandra@rediffmail.com
}

\begin{abstract}
- with increase in threats and concerns in security, detection of suspicious activities in public areas has attracted an enormous level of attention. In general, video processing system are been employed for post-event analysis. However, there is a need to build an intelligent video surveillance system so as to find ways to prevent such events. The proposed system is used todetect the abandoned object from the surveillance videos with the use of dual background model. The division of video into frames is done and are pre-processed. In this approach, dual background method is used to subtract the foreground objects from the background, which generates two backgrounds called buffered background and current background. The foreground blobs are generated using subtraction of the two backgrounds and it is tracked to detect the abandoned objects. Tracking is done by maintaining a Track set which includes blobProperties, namely Area, Centroid, Major Axis length, Minor Axis Length and Convex Area, and two separate count set. The system is tested on various videoswhich are publically available.
\end{abstract}

Keyword - Abandoned object detection, Dual background, Object tracking, Suspicious activity in crowd, Video Surveillance

\section{INTRODUCTION}

Video Surveillance has been an immensely popular tool for security for years. In present days, it is very common to see CCTV cameras located at every public places especially in crowded places like airports, shopping malls, railway stations, movie theatres etc. [19] [20]. Due to the increased crime rate around the world, many organizations are deploying video surveillance systems at their locations with Closed Circuit TV (CCTV) cameras. The captured video data is useful to prevent the threats before the crime actually happens [1]. Video surveillance accounts for regular inspect of the crowd and the security norms can rely on such systems to facilitate their work. However this task remains labour intensive as it become tedious to monitor the entire day footage to detect that one abnormal event. In order to reduce these efforts, intelligent surveillance systems need to be built that can automatically detect if any suspicious activity is encountered and alert the officials whenever required. This provides an efficient and reliable way of monitoring. In video surveillance system, effective and efficient abandoned object detection is one of the important tasks for public security as the bag may contain dangerous bombing components. An object is considered as abandoned if its owner has left it at a place and the object remains stationary for more than an expected time. Considering a scenario where a person enters with an object and places the object onto the floor or a seat in the detection area. The person then leaves the detection area without the object and does not return to the object for a certain pre-determined duration of time [2]. The object continues to remain in the detection area and thus considered as abandoned. These objects are of major concern and needs to be taken care of so as to prevent the hazardous outcome. In order to detect abandoned object from the scene, dual background model is employed. This model comprises two separate backgroundsBuffered and Current Background. An example image is shown in Fig. 1 below showing abandoned object [3].
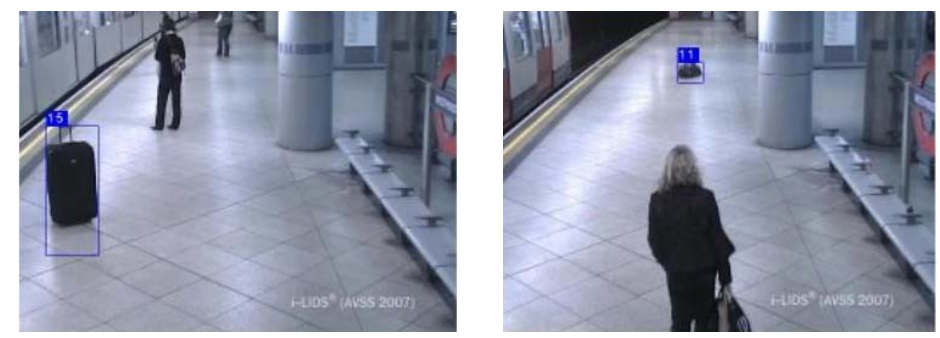

Fig. 1. Example showing abandoned object 


\section{RELATED WORK}

Wahyono, Alexander Filonenko, and Kang-Hyun Jo [3] have presented their work on abandoned objects detection from crowded ccenes of surveillance videos using adaptive dual background model. In this paper, a new framework is presented to detect abandoned object using dual background model subtraction. Major contributions of their work includes: A new background model is introduced based on statistical information of image intensity. Dual background model subtraction is performed in order to extract candidate abandoned region which is robust against lightening changes. Matching-based tracking algorithm is employed to detect abandoned object under occlusion. Human and vehicle detection are integrated to classify human, vehicle and other objects.Quan Wei, Zhang Yuqiang, Ge Wei and LI Hialan [8] have presented their work on research on stationary object detection technique based on dual-Background. A new stationary object detection algorithm is proposed in this paper which includes dual background subtraction to get foreground image based on the approximated median filtering using the adaptive threshold method and detection of stationary object through morphological processing. The target detection algorithm is used the current background and buffer background difference to detect stationary object, then analyse the connected region to abstract the stationary target.Rajesh Kumar Tripathi, Anand Singh Jalal and CharulBhatnagar [10] have presented their work on a framework for abandoned object detection from video surveillance. In this paper,proposed method is used to detect abandoned object from surveillance video. Here, foreground objects are extracted by using background subtraction where background modelling is done through running average method. The objects which are static are detected by using contour features of foreground objects of consecutive frames. Edge based object recognition is used to classify detected static objects into human and non-human objects.A. Singh, S. Sawan, M.Hanmandlu, V.K. Madasu and B.C. Lovell [12] have presented their work on an abandoned object detection system based on dual background segmentation. In this paper, the system is based on a simplistic and intuitive mathematical model. The proposed system consists of a novel self-adaptive dual background subtraction technique based on the approximate median model framework. Tracking is performed on the detected block. A track set is maintained with three variables blobProperties, hitCount and miscount. If hitCount goes above the user defined threshold value, an alarm is triggered indicating the abandoned object is detected. Kevin Lin, Shen-Chi Chen, Chu-Song Chen, Daw-Tung Lin and Yi-Ping Hung [18] have presented their work on abandoned object detection via temporal consistency modelling and back-tracking verification for visual surveillance. Temporal dual-rate foreground integration method is proposed for static-foreground estimation for single camera video images. Subsequently, method introduced a simple pixel-based finite-state machine (PFSM) model that is used to temporal transition information to identify the static foreground based on the sequence pattern of each object pixel. The merits of their system include the dual-rate background modelling framework with temporal consistency which is better than single-image based double background models. It is superior in handling temporary occlusions and is still highly efficient to implement.

\section{III.PROPOSED APPROACH}

In the proposed system, abandoned object detection is carried out from various scenarios. The abstract model of the proposed system is shown in the Fig. 2.

\section{A. Pre-Processing}

A video clip is provided as an input to the system. The input video is then extracted into frames for further processing. Pre-processing is applied on the extracted frames including resizing the video frames then converting frames from RBG to Gray-scale and later applying median filter in order to remove noise and sharpen the edges.

\section{B. Dual Background Generation}

In the proposed approach, Dual Background concept is used rather than the conventionally used simple background subtraction method. In this method, two different backgrounds are maintained- Buffered Background and Current Background. 


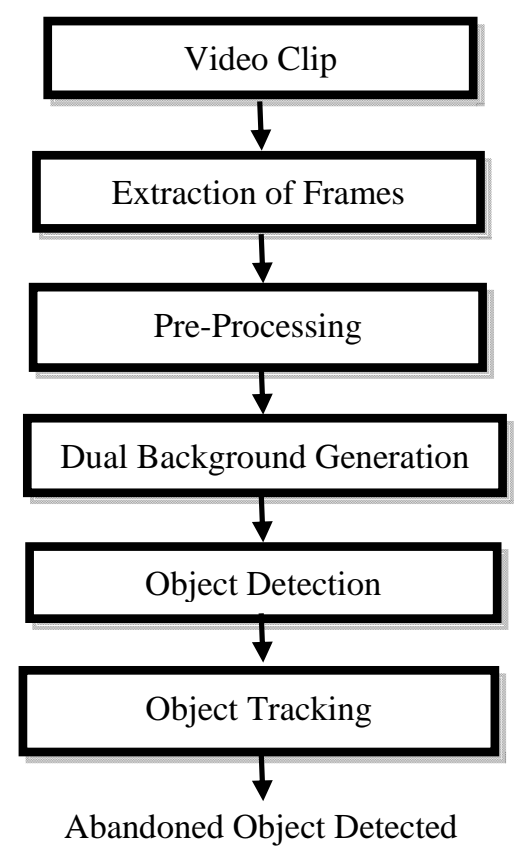

Fig. 2. Abstract Model of the Proposed Approach

The buffered background is initialized by only the first frame of the input video. This background is stored and is not updated. On the other hand, current background is initialized by the first frame and subsequently each pixel of this current background is compared with the corresponding pixel of the next incoming frame. The mathematical model for update strategy is given below:

$$
\mathrm{CB}_{\mathrm{t}+1}=\left\{\begin{array}{l}
\mathrm{CB}_{\mathrm{t}}+1, \text { if } \mathrm{I}_{\mathrm{t}}>\mathrm{CB}_{\mathrm{t}} \\
\mathrm{CB}_{\mathrm{t}}-1, \text { if } \mathrm{I}_{\mathrm{t}}<\mathrm{CB}_{\mathrm{t}} \\
\mathrm{CB}_{\mathrm{t}}, \text { if } \mathrm{I}_{\mathrm{t}}=\mathrm{CB}_{\mathrm{t}}
\end{array}\right.
$$

Where $\mathrm{CB}_{t}$ is the pixel value of current background and $\mathrm{I}_{t}$ is the pixel value of each frame that has been read and t represents time.

\section{Object Detection}

In order to detect the object, difference between the current background and buffer background is calculated after every 10 seconds. The image pixels values are traversed from top to bottom, from left to right. CB(i, j)as current background pixel value and $\mathrm{BB}(\mathrm{i}, \mathrm{j})$ as the buffer background pixel value, then the background subtraction $B(i, j)$ is represented as:

$$
B(i, j)=C B(i, j)-B B(i, j)
$$

After the difference between the two backgrounds is calculated, the result is then binarized depending upon the threshold so as to detect the corresponding suspicious activity. This binarization is shown as follows:

$$
B(i, j)=\left\{\begin{array}{rr}
1, & \text { if }|C B(i, j)-B B(i, j)|>T \\
0, & \text { Otherwise }
\end{array}\right.
$$

Here the value 1 is assigned for those pixels classified as foreground and 0 for those classified as background. Foreground pixels can be grouped into by means of connectivity properties.

\section{Object Tracking}

As from the above step a binary image is obtained, this binary image is divided into number of legitimate blobs i.e., rectangular regions enclosing continuous regions of foreground. Firstly, blobs along with their various properties such as area, centroid, position etc is been generated and later tracking algorithm is applied. Mathematically, it is assumed that after blob analysis $\mathrm{N}$ number of blobs are generated with enclosing region $R_{n}(t, l, h, w)$, having area $A_{n}$, centroid $C_{n}(i, j)$, major axis length $M a_{n}$, minor axis length $\operatorname{Min}_{n}$ and convex area $C A_{n}$, where $t$ is top position value of the pixel, $l$ is the left position value of the pixel, h gives the height of the blob and $\mathrm{w}$ is the width of the blob; and $1 \leq \mathrm{n} \leq \mathrm{N}$. A set of tracked blobs is maintained. ' $\mathrm{T}$ ' is the set of tracked blob defined as,

$$
\mathrm{T}=\left[\mathrm{B}_{\mathrm{n}}: \mathrm{B}_{\mathrm{n}}=\left\{\mathrm{R}_{\mathrm{n}}(\mathrm{t}, \mathrm{l}, \mathrm{h}, \mathrm{w}), \mathrm{A}_{\mathrm{n}}, \mathrm{C}_{\mathrm{n}}(\mathrm{i}, \mathrm{j}), \mathrm{Maj}_{\mathrm{n}}, \operatorname{Min}_{\mathrm{n}}, \mathrm{CA}_{\mathrm{n}}\right\}\right], \forall 1 \leq \mathrm{n} \leq \mathrm{M}
$$


Where, $\mathrm{M}$ is the number of blob tracked. Two count arrays are maintained namely count 1 and count2. Count1 is the set of all individual blobs detected throughout the video frames maintaining total number of times it appeared, centroid values, frame number etc., and count2 is a set maintaining the blobs' records for the current 10 seconds including the blobs that weren't being tracked previously. The new incoming blob detected in count2 is added in the Count1 set if not present already. The next step in object detection is to track the different blobs so as to detect which blob corresponds to abandoned objects. Tracking involves the following steps:

- Create a set, Track, whose elements have six properties: Area, Centroid, Bounding Box, Major axis length, Minor axis length and Convex area collectively called as them blobProperties. This Track set stores properties of all the blobs detected after every 10 seconds interval. Also, two counts sets are maintained.

- For the initial first 10 seconds, add centroid values of each identified blob into the set count1.

- For every interval of 10 seconds analyze the incoming image for all the blobs and store their centroid values into another count set count2. If this set introduces new blobs which are not present in the set count1, then make their entry in count1.

- After 10 seconds is elapsed, comparison is made between the new entries in the Track set with the previous one. The comparison is made to check if all the blobProperties centroid, Area, Major axis length, Minor axis length and Convex area of two blobs are equal or not. If the match is found, the count value is incremented in the count1 set or else the count retained as it is.

- If the value of count reaches a threshold, here 2 indicating 20 seconds, the detected blob is marked with red boundaries indicating it to be potentially abandoned object. These steps are repeated until there are no incoming images.

\section{IV.EXPERIMENTAL RESULTS}

\section{A. Implementation Platform Details}

The hardware and software specifications of the platform on which the proposed approach is implemented and tested is given below:

1) Hardware Specification

- Processor : Intel(R) Core(TM) i3-2370M CPU 2.40GHz

- RAM : $4.00 \mathrm{~GB}$ RAM

2) Software Specification

- OS :Windows 7 Ultimate

- Front End : MATLAB R2012a

\section{B. Tools and Technology}

The whole approach is implemented in MATLAB R2012a. MATLAB is a fourth generation programming language which provides multi-paradigm numerical computing environment. The functions of computer vision system toolbox provide facilities to design and simulate video processing systems. Object detection and tracking, feature extraction and matching, estimation of motion can be done using it.

\section{Dataset Design}

In order to evaluate the presented work, the surveillance video datasets are collected from various resources. The work has been evaluated using 16 video sequences. Video Sequences includes various scenarios: indoor, outdoor, detection in light and crowd. The duration, length, frame rate and scenarios of these video sequences are specified in Table I below. 
TABLE I. Details of Video Sequences Used

\begin{tabular}{|l|l|l|l|l|}
\hline Video Sequence & Duration (minutes) & Length (\#frames) & Frame Rate & \multicolumn{1}{c|}{ Scenario } \\
\hline Video1 & $01: 13$ & 2189 & 29.97 & Outdoor \\
\hline Video2 & $01: 34$ & 2444 & 29.97 & Outdoor \\
\hline Video3 & $01: 27$ & 2608 & 29.97 & Outdoor \\
\hline Video4 & $00: 39$ & 1169 & 29.97 & Outdoor \\
\hline Video5 & $01: 50$ & 3297 & 29.97 & Detection in night \\
\hline Video6 & $01: 22$ & 2485 & 29.97 & Indoor \\
\hline Video7 & $01: 15$ & 2260 & 29.97 & Indoor \\
\hline Video8 & $03: 21$ & 5041 & 25 & Crowded scene \\
\hline Video9 & $02: 00$ & 3021 & 25 & Crowded scene \\
\hline Video10 & $01: 42$ & 2551 & 25 & Crowded scene \\
\hline Video11 & $01: 34$ & 2371 & 25 & Crowded scene \\
\hline Video12 & $01: 34$ & 2371 & 25 & Crowded scene \\
\hline Video13 & $02: 02$ & 3051 & 25 & Crowded scene \\
\hline Video14 & $02: 02$ & 3051 & 25 & Crowded scene \\
\hline Video15 & $02: 16$ & 3401 & 25 & Crowded scene \\
\hline Video16 & $01: 52$ & 2801 & 25 & Crowded scene \\
\hline
\end{tabular}

\section{Experimental Results}

The video1 video sequence is chosen for testing which contains outdoor scenario. The duration of the video is 73 seconds having 2189 frames with frame rate is 29.97. As per the ground truth, 1 abandoned object is present in the video. The first phase of the system includes: Extraction of first frame, resize it, convert from RGB to Gray and initialize it to Buffered and Current Background.

The next phase is to run the video in segments of size 10 seconds. Here since the duration of video is 73 seconds thus the segments obtained are 8. Frame extraction is carried out and the pixel values of next incoming video frame are compared with that of the current background for the each segment i.e., for the every 10 seconds of the video. Figure 3 shows the experimental result for the last segment as we detect abandoned object in that segment.Fig. 3(a) shows the buffered background, Fig. 3(b) shows the updated current background and Fig. 3(c) shows the binarized difference image.

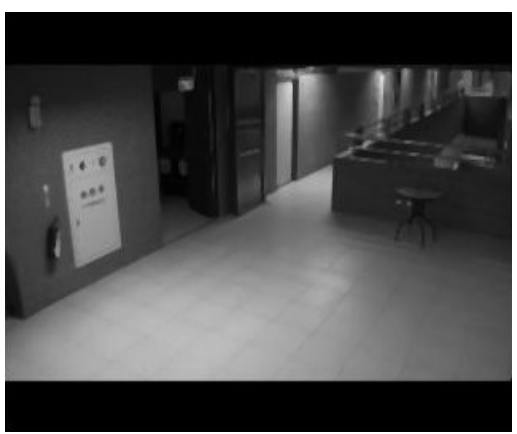

(a)

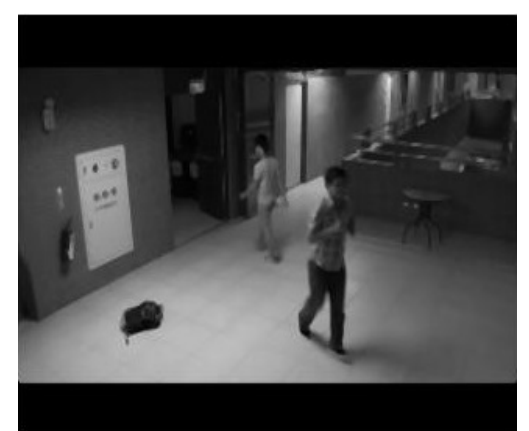

(b)

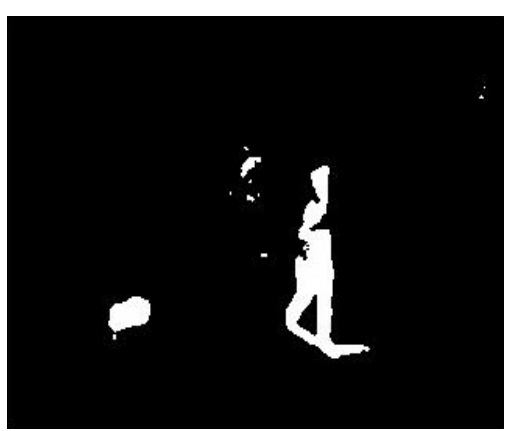

(c)

Fig. 3. Dual background subtractions for segment 8

Here, three blobs are obtained whose blobProperties are listed in the Table II below:

TABLE II. BlobProperties for Segment 8

\begin{tabular}{|l|l|c|l|l|l|l|}
\hline & Area & Bounding Box & Centroid & $\begin{array}{c}\text { Major Axis } \\
\text { Length }\end{array}$ & $\begin{array}{c}\text { Minor axis } \\
\text { Length }\end{array}$ & $\begin{array}{c}\text { Convex } \\
\text { Area }\end{array}$ \\
\hline Blob1 & 397 & {$[66.5,163.5,27,21]$} & {$[80.16,173.56]$} & 29.68 & 17.44 & 427 \\
\hline Blob2 & 71 & {$[151.5,82.5,13,12]$} & {$[157.07,87.09]$} & 16.50 & 6.59 & 85 \\
\hline Blob3 & 1889 & {$[180.5,87.5,54,112]$} & {$[200.44,151.44]$} & 116.65 & 35.19 & 3672 \\
\hline
\end{tabular}


The values in array count 2 are updated as shown in the Table III below:

TABLE III. Count2 Set for Segment 8

\begin{tabular}{|l|l|}
\hline \multicolumn{1}{|c|}{ Centroid $\mathbf{x}$-axis } & \multicolumn{1}{c|}{ Centroid $\mathbf{y}$-axis } \\
\hline 80.1587 & 173.5617 \\
\hline 157.0704 & 87.0986 \\
\hline 200.4436 & 151.4426 \\
\hline
\end{tabular}

Now here, the centroid of blob1, blob2 and blob3 matches with the centroid of 67th, 70th and 71th entries in the set count1 respectively. Thus their values are overwritten by these new values. No new entries are included in count1. Below is Table IV showing the updated count1 set from 67th position.

TABLE IIV. Count1 Set after Segment 8

\begin{tabular}{|l|l|l|}
\hline Count & \multicolumn{1}{|c|}{ Centroid $x$-axis } & \multicolumn{1}{c|}{ Centroid y-axis } \\
\hline 1 & 80.1587 & 173.5617 \\
\hline 0 & 165.9322 & 126.5403 \\
\hline 0 & 172.5351 & 94.5789 \\
\hline 0 & 157.0704 & 87.0986 \\
\hline 0 & 200.4436 & 151.4426 \\
\hline
\end{tabular}

The comparison between the blobProperties of recently obtained blobs is made with the ones obtained in the previous segment. Comparison of the three blobs is shown is shown in the Table V along with their respective matches from the previous segment.

TABLE V. Comparison of Blobs between Segment 7 and Segment 8

\begin{tabular}{|l|l|l|l|l|l|}
\hline & Area & \multicolumn{1}{|c|}{ Centroid } & \multicolumn{1}{|c|}{$\begin{array}{c}\text { Major Axis } \\
\text { Length }\end{array}$} & $\begin{array}{c}\text { Minor } \\
\text { Axis Length }\end{array}$ & $\begin{array}{c}\text { Convex } \\
\text { Area }\end{array}$ \\
\hline Blob1(Segment7) & 402 & {$[80.1070,173.5149]$} & 29.8497 & 17.5055 & 429 \\
\hline Blob1(Segment8) & 397 & {$[80.1587,173.5617]$} & 29.6758 & 17.4411 & 427 \\
\hline Blob2(Segment7) & 71 & {$[151.5,82.5,13,12]$} & {$[157.07,87.09]$} & 16.50 & 6.59 \\
\hline Blob2(Segment8) & 71 & {$[151.5,82.5,13,12]$} & {$[157.07,87.09]$} & 16.50 & 6.59 \\
\hline Blob3(Segment7) & 1889 & {$[180.5,87.5,54,112]$} & {$[200.44,151.44]$} & 116.65 & 35.19 \\
\hline Blob3(Segment8) & 1889 & {$[180.5,87.5,54,112]$} & {$[200.44,151.44]$} & 116.65 & 35.19 \\
\hline
\end{tabular}

Here, it is observed that all the three blobs in this segment match with previously obtained blobs in terms of area, centroid, major axis length, minor axis length and convex area. Thus the count of blob1 in set count1 is incremented by 1 making it a total of 2 . Similarly, count of blob2 and blob3 are also incremented by 1 . The Table VI is shown below with the incremented values of the count:

TABLE VI. Updated Count1 Set after Completion of the Video1

\begin{tabular}{|l|l|l|}
\hline Count & \multicolumn{1}{|c|}{ Centroid $\mathbf{x}$-axis } & \multicolumn{1}{c|}{ Centroid y-axis } \\
\hline 2 & 80.1587 & 173.5617 \\
\hline 0 & 165.9322 & 126.5403 \\
\hline 0 & 172.5351 & 94.5789 \\
\hline 1 & 157.0704 & 87.0986 \\
\hline 1 & 200.4436 & 151.4426 \\
\hline
\end{tabular}

The threshold set in the proposed system to label the object as abandoned is 20 seconds. Here, the count of value 2 indicates 20 seconds, meaning that the object is left abandoned for 20 seconds. Once the object is detected as abandoned, the boundary surrounding the object is changed to red colour. Figure 4 shows the three identified objects with two marked with green bounding box while one marked with red bounding box, indicating it to be abandoned. 


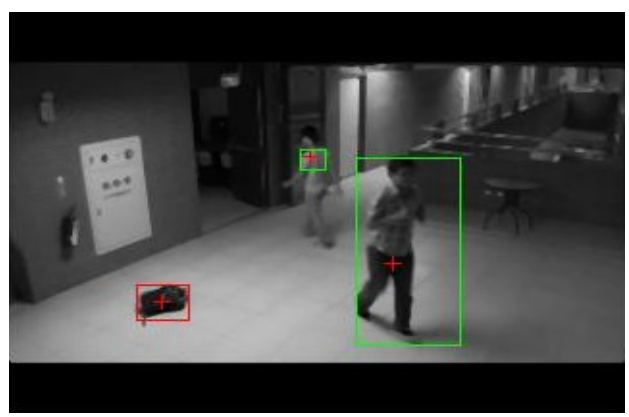

Fig. 4. Abandoned object detected

Experiments are performed on various other video sequences including outdoor, indoor, detection in night and crowded scenes. Table VII shows the summary of the detection results on outdoor, indoor and detection in night. Here, total of 7 videos are used. Ground truth is provided with every video having one abandoned object.

TABLE VII. Detection Results on Outdoor, Indoor and in Night Scenarios

\begin{tabular}{|l|l|l|l|l|l|l|}
\hline Video Sequence & GT & TN & TP & FN & FP & Scenario \\
\hline Video1 & 1 & 0 & 1 & 0 & 0 & Outdoor \\
\hline Video2 & 1 & 0 & 1 & 0 & 0 & Outdoor \\
\hline Video3 & 1 & 0 & 1 & 0 & 0 & Outdoor \\
\hline Video4 & 1 & 0 & 1 & 0 & 0 & Outdoor \\
\hline Video5 & 1 & 0 & 1 & 0 & 2 & Detection in night \\
\hline Video6 & 1 & 0 & 1 & 0 & 0 & Indoor \\
\hline Video7 & 1 & 0 & 1 & 0 & 0 & Indoor \\
\hline
\end{tabular}

Here, GT $=$ Ground Truth, $\mathrm{TN}=$ True Negative, $\mathrm{TP}=$ True Positive, $\mathrm{FN}=$ False Negative and FP $=$ False Positive. The detection results from the above table shows that the abandoned objects are successfully detected for all the video sequences with precision of $77.78 \%$ and recall being $100 \%$. Only two false positives are obtained from video sequence 5 which is mainly due to unclean buffered background.Similarly, experiment performed on crowded scenario is shown in the below Table VIII. Here total of 9 videos are used each having crowded scene. Ground truth is provided with every video containing one abandoned object except for videos 4 and 5, having no abandoned object.

TABLE VIII. Detection Results for Crowded Scenario

\begin{tabular}{|l|l|l|l|l|l|l|}
\hline Video Sequence & GT & TN & TP & FN & FP & Scenario \\
\hline Video1 & 1 & 0 & 1 & 0 & 1 & Crowded scene \\
\hline Video2 & 1 & 0 & 1 & 0 & 0 & Crowded scene \\
\hline Video3 & 1 & 0 & 1 & 0 & 1 & Crowded scene \\
\hline Video4 & 0 & 1 & 0 & 0 & 1 & Crowded scene \\
\hline Video5 & 0 & 1 & 0 & 0 & 0 & Crowded scene \\
\hline Video6 & 1 & 0 & 1 & 0 & 1 & Crowded scene \\
\hline Video7 & 1 & 0 & 1 & 0 & 1 & Crowded scene \\
\hline Video8 & 1 & 0 & 1 & 0 & 0 & Crowded scene \\
\hline Video9 & 1 & 0 & 1 & 0 & 1 & Crowded scene \\
\hline
\end{tabular}

From the above table it is observed that all the abandoned objects are accuratelydetected but also few false positives are detected because of the crowded nature of the videos and unclear first frame resulting in the unclean buffered background. The precision observed is $53.85 \%$ while recall remains $100 \%$. Combining all the video sequences, the overall precision is calculated to be $63.63 \%$ and the recall is $100 \%$. 


\section{CONCLUSION AND FUTURE WORK}

This paper presents an approach based on dual background model which detects abandoned object from the surveillance videos. Dual background model maintains two separate backgrounds one called buffered background and other called current background.Experiments are performed on various video sequences for different scenarios including indoor, outdoor, detection in night and crowded scenes. The experimental results for video sequences having outdoor, indoor and detection in night scenario has precision of $77.78 \%$ and recall of $100 \%$. For crowded scenario the precision measured is $53.85 \%$ and the recall measured is $100 \%$. The overall precision for all the video sequences is measured to be $63.63 \%$ with $100 \%$ recall.Though the proposed system works well but in future it can be extended to work better even in densely crowded places. Occlusion of the objects continues to be an issue, which can be solved if way to update the buffered background is improved, thus enhancing the object detection by the system.

\section{REFERENCES}

[1] SandeshPatil and KiranTalele, "Suspicious movement detection and tracking based on color histogram.” International Conference on Communication, Information and Computing Technology (ICCICT), pp.1-6, 2015.

[2] Home Office Scientific Development Branch, "Imagery Library for Intelligent Detection Systems (i-LIDS); A Standard for Testing Video Based Detection Systems”, Proceedings 40th Annual International Carnahan Conference on Security Technology, pp. 75-80, 2006.

[3] Wahyono, Alexander Filonenko, and Kang-Hyun Jo, "Detecting Abandoned Objects in Crowded Scenes of Surveillance Videos using Adaptive Dual Background Model” 8th International Conference on Human System Interaction (HSI), IEEE, pp. 224-227, 2015.

[4] Tobias Senst, Volker Eiselein, Ivo Keller and Thomas Sikora, "Crowd analysis in non-static cameras using feature tracking and multiperson density", IEEE International Conference on Image Processing (ICIP). IEEE, pp. 6041-6045, 2014.

[5] Dongliang Jin, Songhao Zhu, Xian Sun, Zhiwei Liang and GuozhengXu, "Optical Flow and Spatio-temporal Gradient Based Abnormal Behavior Detection”Chinese Control and Decision Conference (CCDC), IEEE, pp. 1532-1537, 2016.

[6] Ang Li, Zhenjiang Miao, Yigang Cen and Qinghua Liang, "Abnormal Event Detection based on Sparse Reconstruction in Crowded Scenes”, IEEE International Conference on Acoustics, Speech and Signal Processing (ICASSP), pp. 1786-1790, 2016.

[7] Rubén HerasEvangelio, Michael Patzold and Thomas Sikora, "Static object detection based on a dual background model and a finitestate machine" EURASIP Journal on Image and Video Processing, 2011.

[8] Quan Wei, Zhang Yuqiang, Ge Wei and LI Hialan, "Research on Stationary Object Detection Technique based on Dual-Background”, Fifth International Conference on Instrumentation and Measurement, Computer, Communication and Control (IMCCC), IEEE, pp. 1783-1786, 2015.

[9] Nathaniel Bird, Stefan Atev, Nicolas Caramelli, Robert Martin, Osama Masoud and NikolaosPapanikolopoulos, "Real Time Online Detection of Abandoned Objects in Public Areas", Proceedings 2006 IEEE International Conference on Robotics and Automation, ICRA, IEEE, pp. 3775-3780, 2006.

[10] Rajesh Kumar Tripathi, Anand Singh Jalal and CharulBhatnagar, "A Framework for Abandoned Object Detection from Video Surveillance”, Computer Vision, Pattern Recognition, Image Processing and Graphics (NCVPRIPG), Fourth National Conference, IEEE, pp. 1-4, 2013.

[11] Rajesh Rohilla, Aman Raj, SaranshKejriwal and Rajiv Kapoor, "FPGA accelerated abandoned object detection”, International Conference on Computational Techniques in Information and Communication Technologies (ICCTICT), pp. 302-306, 2016.

[12] A. Singh, S. Sawan, M. Hanmandlu, V.K. Madasu and B.C. Lovell, "An abandoned object detection system based on dual background segmentation”, Advanced Video and Signal Based Surveillance, Sixth IEEE International Conference, IEEE, pp. 352-357, 2009.

[13] Luis Caro Campos, Juan CarlosSanMiguel and Jose M. Martinez, "Discrimination of Abandoned and Stolen Objects based on Active Contours”, 8th IEEE International Conference on Advanced Video and Signal-Based Surveillance (AVSS), pp. 101-106, 2011.

[14] Jiyan Pan, Quanfu Fan and SharathPankanti, "Robust Abandoned Object Detection using Region-Level Analysis”, 18th IEEE International Conference on Image Processing, IEEE, pp. 3597-3600, 2011.

[15] YingLiTian, Rogerio Schmidt Feris, Haowei Liu, ArunHampapur and Ming-Ting Sun, "Robust Detection of Abandoned and Removed Objects in Complex Surveillance Videos”, IEEE Transactions on Systems, Man, and Cybernetics, Part C (Applications and Reviews), Volume: 41, Issue: 5, pp. 565-576, 2011.

[16] Kahlil Muchtar, Chih-Yanh Lin and Chia-Hung Yeh, “GrabCut- Based Abandoned Object Detection”, IEEE 16th International Workshop on Multimedia Signal Processing (MMSP), pp. 1-6, 2014.

[17] DeigoOrtego, Juan C. SanMiguel and Jose M. Martinez, "Long-Term Stationary object detection based on spatio-temporal change detection”, IEEE Signal Processing Letters, Volume: 22, Issue: 12, pp. 2368-2372, 2015.

[18] Kevin Lin, Shen-Chi Chen, Chu-Song Chen,Daw-Tung Lin and Yi-Ping Hung, "Abandoned Object Detection via Temporal Consistency modeling and Back-Tracking verification for Visual Surveillance”, IEEE Transactions on Information Forensics and Security, Volume: 10, Issue: 7, pp. 1359-1370, 2015.

[19] Dhamsania, Chandni, and Tushar Ratanpara. "Human Action Recognition Using Trajectory-Based Spatiotemporal Descriptors." Proceedings of the 5th International Conference on Frontiers in Intelligent Computing: Theory and Applications. Springer, Singapore, 2017.

[20] ChandniDhamsania, and Tushar Ratanpara "A survey on Human action recognition from videos." International Conference on Green Engineering and Technologies (IC-GET), pp. 1-5, 2016.

\section{AUTHOR PROFILE}

Purvi Bhandari has completed Master of Technology in computer engineering from Dharmsinh Desai University, Nadiad, India. Her main area of interest is in image and video processing.

Prof. Tushar Ratanpara is working as an Assistant Professor in computer engineering department at Dharmsinh Desai University, Nadiad, India. He is also working as a counselor of CSI-DDU student branch since 2011. He has published more than 15 research papers in the international journals and conferences like IEEE, Springer, IGI-Global, Inderscience and ACM. His main research areas includes Image Processing, Video Processing, Audio Processing and Internet of Things. 
Dr. K. H. Wandra has completed Ph.D. from Saurashtra University, Rajkot. He is currently working as aDean of Faculty of Technology and Engineering \& Principal, C. U. Shah College of Engineering \& Technology, Gujarat, India. He was a Chairman of Board of Studies Computer Engineering at Saurashtra University, Rajkot, Core Committee member For Syllabus of UG \& PG at Gujarat Technology University, Ahmedabad Section Managing Committee Member of ISTE Gujarat Section, Life member of ISTE, member of IEEE and CSI, Interested for working in the area of Wireless communication, Networks, Computer Vision, Video Processing and Advanced Microprocessors. 